\title{
CORRESPONDENCE \\ Epitopes for a 2019-nCoV vaccine
}

\author{
Guglielmo Lucchese (iD) \\ Cellular \& Molecular Immunology (2020) 17:539-540; https://doi.org/10.1038/s41423-020-0377-z
}

After causing an initial cluster of Pneumonia in Wuhan City, Hubei Province, the 2019-nCoV has quickly spread through South East Asia and within a few weeks to Europe, Africa, and America. Initial estimates suggested a mortality rate of $2 \%$ and that $\sim 18 \%$ of the cases show severe symptoms, although such estimates are still subject to rapid changes (https://www.who.int/news-room/detail/ 30-01-2020-statement-on-the-second-meeting-of-the-internationalhealth-regulations-(2005)-emergency-committee-regarding-theoutbreak-of-novel-coronavirus-(2019-ncov)). ${ }^{1-3}$

To facilitate the swift development of a candidate vaccine for 2019-nCoV we compared here the viral and the human proteomes, searching for pentapeptides that are unique to the pathogen. We followed the rationale that non-self sequences are highly immunogenic and uniquely viral epitopes should improve safety and efficacy by minimizing the risk for cross-reactions and increasing anti-viral specificity. ${ }^{4-6}$ The analysis was conducted on the entire viral proteome but primarily focused on the surface spike glycoprotein (id = "QHD43416.1) because immune response against it is highly likely to exert a neutralizing effect. ${ }^{2}$

The entire amino acid (aa) sequence of the 2019-nCoV was retrieved from https://www.ncbi.nlm.nih.gov/nuccore/MN908947 and dissected into pentapeptides overlapped by four residues for a total of $n=9661$. Then, each pentamer was analyzed for occurrences in the human proteome using the Peptide Match program (https://research.bioinformatics.udel.edu/peptidematch/ index.jsp). $^{7}$

It resulted that $n=933$ viral pentapeptides are absent in the human proteome, and therefore foreign to the human immune system (Table S1). Among these non-self pentapeptides, $n=107$ are embedded in the viral surface glycoprotein (spike protein) that mediates binding to the human ACE2 and cellular entry. ${ }^{2}$

The recommended oligopeptides for a multi-epitope 2109nCoV-vaccine are presented in Table 1, Panel a. They can be rapidly tested in animal models for immunogenicity and safety in order to timely develop a vaccine for preventing uncontrolled spreading of the novel coronavirus.

Three points need to be stressed.

First, short peptides that are foreign to the host immune system have been experimentally validated not only as positive immunomodulants (i.e., adjuvants) in conjunction to vaccines, but are also evidenced as providing direct protection against lethal viral infections, at least in animal models. ${ }^{6}$

Second, searching for the 107 human-foreign spike protein pentapeptides in the Immune Epitope Database (IEDB; www.iedb. org) ${ }^{8}$ yielded a list of $n=66$ epitopes (Table 1, Panel b). The IEDB is a publicly available, curated epitope repository. The presence of a peptide sequence in the IEDB indicates that it has a recognized and experimentally proven immunologic relevance. These results provide experimental proof for the immunogenic potential of the non-self peptides identified in the present study through comparative Homo sapiens-coronavirus proteome analysis.

Table 1. (a) Oligopeptides $(n=73)$ of the spike protein absent in the human proteome to be tested for a potential vaccine. Contiguous pentapeptides with a four residue overlap were considered as a single, longer oligopeptidic sequence; the length of each of these oligopeptides was dictated by the extension of the overlap. Oligopeptides from epitopes in panel $\mathrm{b}$ are in bold. (b) Experimentally validated epitopes $(n=66)$ containing at least one of the 107 pentapeptides (capitalized) of the spike protein that are absent in the human proteome

(a) RGVYYPDK, NVTWFHA, FHAIH, PFNDG, IRGWIF, IFGTT, VCEFQFC, CNDPF, VYYHK, NNKSW, NKSWM, WMESEF, YSSAN, CTFEY, GNFKN, GYFKI, IYSKHT, PIGIN, GWTAG, AYYVG, NENGT, SETKC, GIYQT, VYAWNR, CVADY, STFKC, FKCYGVS, TNVYA, IADYN, DYNYKL, VIAWN, AWNSNN, STPCN, PCNGV, GFNCYF, QSYGF, VKNKC, NKCVN, CVNFN, CTEVP, IGAEH, YOTOTN, IAYTMS, TSVDC, DCTMY, TMYICG, DSTEC, FCTOL, PIKDF, OYGDCL, GDCLG, YQTQTN, IAYTMS, TSVDC, DCTMY, TMYICG, DSTEC, FCTQL, PIKDF, QYGDCL, GDCLG, PAICH, NGTHWFVTQ, TQRNF, NFYEP, IGIVN, NTVYD, IKWPWYI, YIWLGF, IAIVM, LCCMTS, MTSCC, CCKFD

(b) IEDB-ID-Number 307 462

1460

3176

6011

6333

6334

7066

7066
7217

7383

8239

12417

15903

18161

18515

21464

22321

24978

25250

25293

25378

25382

29728

30987

30988

31581

31582

33305
Epitope

aalvsgtatagWTFGAg

aatkMSECVlgqskrvd

agclIGAEHvdtsyecd

aMQMAYRF

canlllqygsFCTQLnralsgia

cgpklstdliknqCVNFNfngltgtgvltpsskrfqpfqqfg

cgpklstdliknqCVNFNfngltgtgvltpsskrfqpfqqfgrdvsdftd

csqnplaelkcsvksfeidkGIYQTsnfrvvpsgd

cttfddvqapnytqhtssmRGVYYPDeifr

CYGVSatkIndlcfsnv

eidkGIYQTsnfrvvps

ffSTFKCYGVSatklnd

fvfngtswfiTQRNFfs

gaalqipFAMQMAYRFn

gnliaprGYFKIrsgkssim

gsFCTQLn

htssmRGVYYPDeifrs

IADYNYKLpddfmgcvl

iagllAIVMvtillccm

iapgqtgvIADYNYKLp

iywtivkpgdillinstgnliaprGYFKIrn

kGIYQTsn

kGIYQTsnfrvvpsgdvvrf

kkisnCVADYsvlynst

kkisnCVADYsvlynstf

ksfeidkGIYQTsnfrvv
dfcgkGYHLMSfpqaap

iaprGYFKIrngkssimrsdapigtcssecit

${ }^{1}$ Universitätsmedizin Greifswald, Department of Neurology, 17475 Greifswald, Germany

Correspondence: Guglielmo Lucchese (guglielmo.lucchese@uni-greifswald.de)

Received: 10 February 2020 Accepted: 11 February 2020

Published online: 24 February 2020 


\begin{tabular}{|c|c|}
\hline $\begin{array}{l}33358 \\
33874\end{array}$ & $\begin{array}{l}\text { ksivAYTMSIgadssia } \\
\text { KTSVDCnMYICGDSTEC }\end{array}$ \\
\hline 36579 & liknqCVNFNfngltgt \\
\hline 36815 & IkcsvksfeidkGIYQT \\
\hline 36856 & IkgacscgsCCKFDedd \\
\hline 37758 & IlrstsqksivAYTMSI \\
\hline 39023 & laygsFCTQLnralsgi \\
\hline 41177 & MAYRFNGlgvtqnvlye \\
\hline 42999 & mvtilLCCMTSCCsclk \\
\hline 43145 & nafnCTFEYisdafsld \\
\hline 46379 & nvfqtqagclIGAEHvd \\
\hline 46822 & PAICHegkayfpregvfufngtswfitgrnffs \\
\hline 47479 & pFAMQMAYRFNGlgvtq \\
\hline 49968 & pvsmakTSVDCnMYICGds \\
\hline 50058 & pwyvwlgfiagliAIVM \\
\hline 53202 & rasanlaatkMSECVIg \\
\hline 54989 & rnfttaPAICHegkayf \\
\hline 58143 & sgncdvvigiinNTVYD \\
\hline 58730 & SivAYTMSI \\
\hline 61554 & stdliknqCVNFNfn \\
\hline 61598 & stffSTFKCYGVSatkl \\
\hline 62872 & tagWTFGAgaalqipfa \\
\hline 63309 & tecanlllqygsFCTQL \\
\hline 68971 & vigiinNTVYDplqpel \\
\hline 72205 & VYYPDeifrsdtlyltad \\
\hline 74173 & yicgDSTECanlllqyg \\
\hline 75920 & ysvlynstffSTFKCYG \\
\hline 99918 & CTFEYisdafsld \\
\hline 100048 & gaalqipFAMQMAYRF \\
\hline 100230 & ksivAYTMSlgadssiay \\
\hline 100300 & MAYRFNGlgvtqnuly \\
\hline 100316 & nafnCTFEYisdafsldv \\
\hline 100537 & swfiTQRNFfspqii \\
\hline 100711 & agcllGAEHvdtsyecdi \\
\hline 129239 & liaprGYFKIrsgkssi \\
\hline 532052 & gtswfiTQRNFfspq \\
\hline 873061 & mmcehiyytcvrTSVDCc \\
\hline 874104 & ytcvrTSVDCcmkgaep \\
\hline
\end{tabular}

Third, an immune response induced by the spike protein oligopeptides that are absent in the human proteins would exert a neutralizing effect on the coronavirus, in light of the mounting evidence for the surface glycoprotein as a ligand for the human ACE2 in viral entry processes. ${ }^{2}$

\section{ACKNOWLEDGEMENTS}

The author is supported by a "Gerhard Domagk" research grant awarded by the University of Greifswald Medical School.

\section{ADDITIONAL INFORMATION}

The online version of this article (https://doi.org/10.1038/s41423-020-0377-z) contains supplementary material.

Competing interests: The author declares no competing interests.

\section{REFERENCES}

1. Zhu, N. et al. A novel coronavirus from patients with pneumonia in China. N. Engl. J. Med. https://doi.org/10.1056/NEJMoa2001017 (2019).

2. Gralinski, L. E. \& Menachery, V. D. Return of the Coronavirus: 2019-nCoV. Viruses 12.2, 135 (2020).

3. Huang, C. et al. Clinical features of patients infected with 2019 novel coronavirus in Wuhan, China. Lancet 395, 497-506 (2020).

4. Richman, L. P., Vonderheide, R. H. \& Rech, A. J. "Neoantigen dissimilarity to the selfproteome predicts immunogenicity and response to immune checkpoint blockade." Cell Syst. 9.4, 375-382 (2019).

5. Kanduc, D. in Multichain Immune Recognition Receptor Signaling. Advances in Experimental Medicine and Biology (ed. Sigalov, A. B.) 640 (Springer, New York, NY, 2008).

6. Patel, A. et al. Pentamers not found in the universal proteome can enhance antigen specific immune responses and adjuvant vaccines. PLOS ONE 7, e43802, https://doi.org/10.1371/journal.pone.0043802 (2012).

7. Chen, C., Li, Z., Huang, H., Suzek, B. E. \& Wu, C. H. UniProt Consortium. A fast Peptide Match service for UniProt Knowledgebase. Bioinformatics 29, 2808-2809 (2013).

8. Vita, R. et al. The immune epitope database 2.0. Nucleic Acids Res. 38, D854-D862 (2010).

Open Access This article is licensed under a Creative Commons cc) Attribution 4.0 International License, which permits use, sharing, adaptation, distribution and reproduction in any medium or format, as long as you give appropriate credit to the original author(s) and the source, provide a link to the Creative Commons license, and indicate if changes were made. The images or other third party material in this article are included in the article's Creative Commons license, unless indicated otherwise in a credit line to the material. If material is not included in the article's Creative Commons license and your intended use is not permitted by statutory regulation or exceeds the permitted use, you will need to obtain permission directly from the copyright holder. To view a copy of this license, visit http://creativecommons. org/licenses/by/4.0/.

(c) The Author(s) 2020 